\title{
General Psychiatry High levels of psychosocial distress among Australian frontline healthcare workers during the COVID-19 pandemic: a cross-sectional survey
}

\author{
Natasha Smallwood, ${ }^{1,2}$ Leila Karimi, ${ }^{3,4}$ Marie Bismark, ${ }^{5,6}$ Mark Putland (D) ,7,8 \\ Douglas Johnson, ${ }^{9,10}$ Shyamali Chandrika Dharmage, ${ }^{11}$ Elizabeth Barson, ${ }^{12}$ \\ Nicola Atkin, ${ }^{13,14}$ Claire Long, ${ }^{15}$ Irene $\mathrm{Ng},{ }^{16,17}$ Anne Holland, ${ }^{2,18}$ Jane E Munro, ${ }^{19,20}$ \\ Irani Thevarajan, ${ }^{21}$ Cara Moore, ${ }^{22}$ Anthony McGillion, ${ }^{23}$ Debra Sandford, ${ }^{24}$ \\ Karen Willis ${ }^{25,26}$
}

To cite: Smallwood N, Karimi L, Bismark M, et al. High levels of psychosocial distress among Australian frontline healthcare workers during the COVID-19 pandemic: a cross-sectional survey. General Psychiatry 2021;34:e100577. doi:10.1136/ gpsych-2021-100577

- Additional supplemental material is published online only. To view, please visit the journal online (http://dx.doi.org/10.1136/ gpsych-2021-100577).

Received 20 May 2021 Accepted 03 August 2021

A) Check for updates

(c) Author(s) (or their employer(s)) 2021. Re-use permitted under CC BY-NC. No commercial re-use. See rights and permissions. Published by BMJ.

For numbered affiliations see end of article.

Correspondence to Dr Natasha Smallwood; natasha.smallwood@monash. edu.au

\section{ABSTRACT}

Background The coronavirus disease 2019 (COVID-19) pandemic has had a profound and prolonged impact on healthcare services and healthcare workers.

Aims The Australian COVID-19 Frontline Healthcare Workers Study aimed to investigate the severity and prevalence of mental health issues, as well as the social, workplace and financial disruptions experienced by Australian healthcare workers during the COVID-19 pandemic.

Methods A nationwide, voluntary, anonymous, single timepoint, online survey was conducted between 27 August and 23 October 2020. Individuals self-identifying as frontline healthcare workers in secondary or primary care were invited to participate. Participants were recruited through health organisations, professional associations or colleges, universities, government contacts and national media. Demographics, home and work situation, health and psychological well-being data were collected.

Results A total of 9518 survey responses were received; of the 9518 participants, 7846 (82.4\%) participants reported complete data. With regard to age, $4110(52.4 \%)$ participants were younger than 40 years; 6344 (80.9\%) participants were women. Participants were nurses $(n=3088,39.4 \%)$, doctors $(n=2436,31.1 \%)$, allied health staff $(n=1314$, $16.7 \%$ ) or in other roles $(n=523,6.7 \%)$. In addition, 1250 (15.9\%) participants worked in primary care. Objectively measured mental health symptoms were common: mild to severe anxiety ( $n=4694,59.8 \%)$, moderate to severe burnout ( $\mathrm{n}=5458,70.9 \%$ ) and mild to severe depression $(n=4495,57.3 \%)$. Participants were highly resilient (mean $(S D)=3.2(0.66))$. Predictors for worse outcomes on all scales included female gender; younger age; pre-existing psychiatric condition; experiencing relationship problems; nursing, allied health or other roles; frontline area; being worried about being blamed by colleagues and working with patients with COVID-19.

Conclusions The COVID-19 pandemic is associated with significant mental health symptoms in frontline healthcare workers. Crisis preparedness together with policies and practices addressing psychological well-being are needed.

\begin{tabular}{|l} 
KEY MESSAGES \\
\hline THE KNOWN \\
\hline Healthcare workers experience unique workplace \\
demands and stressors in their day-to-day roles and \\
therefore are at increased risk of mental illness. \\
$\Rightarrow$ Crisis events represent an additional threat to men- \\
tal health of healthcare workers. \\
$\Rightarrow$ Poor mental health of clinicians has wider repercus- \\
sions for quality of care, patient safety, workforce \\
retention and engagement. \\
THE NEW \\
\hline$\Rightarrow$ The impacts of the COVID-19 pandemic were asso- \\
ciated with significant symptoms of mental illness \\
in Australian frontline healthcare workers in primary \\
and secondary care. \\
$\Rightarrow$ Some healthcare worker groups were more vulner- \\
able to psychological problems. \\
$\Rightarrow$ Health organisations and leaders need to be better \\
prepared for crisis events such as pandemics, given \\
the associated impacts observed in mental health \\
of frontline healthcare workers. Additional psycho- \\
logical well-being services are needed to actively \\
support healthcare workers.
\end{tabular}

\section{INTRODUCTION}

Healthcare workers (HCWs) experience unique workplace demands and stressors, with doctors and nurses particularly experiencing poor mental health and increased rates of occupational burnout, anxiety, depression and suicide than other occupations. ${ }^{1-4}$ Although less is known about other groups of clinicians, the findings of early studies are concerning. ${ }^{5}$ These issues have ramifications beyond the health of practitioners themselves, given that poor mental health of clinicians translates to adverse effects on overall 
quality of care, ${ }^{6}$ patient safety, workforce retention and engagement. ${ }^{7}$

Crises, such as the current coronavirus disease 2019 (COVID-19) pandemic, represent a profound threat to mental health. HCWs, particularly those on the 'frontline' in hospitals and the community, have had to respond quickly to many challenges including heavy workloads, large volumes of new information, new work practices and roles, redeployment or job insecurity, social change and increased risks to their own lives and for family members. Evidence regarding the impacts of the severe acute respiratory syndrome (SARS) pandemic demonstrated that the mental health of many HCWs was adversely affected, with potentially long-lasting mental health effects. ${ }^{8}$ Studies from overseas during the current pandemic suggest high rates of anxiety, depression, stress and burnout in HCWs, with the prevalence rates of up to $57 \% .^{10-16}$ Before the onset of COVID-19, certain demographic and workplace factors have been associated with increased risk of psychosocial harm for HCWs, particularly female gender, ${ }^{2}$ inexperience ${ }^{17}$ excessive work hours, ${ }^{18} 19$ and certain frontline areas. ${ }^{20}{ }^{21}$ Similarly, studies of the general public during COVID-19 reveal a disproportionate impact on women,${ }^{22}{ }^{23}$ young people ${ }^{22}{ }^{24}$ and people with previous mental health diagnoses. ${ }^{24}{ }^{25}$ It is therefore vital to comprehensively identify and act on the mental health needs of Australian frontline HCWs to minimise the far-reaching effects of crisis events. This article reports the first findings from the Australian COVID-19 Frontline Healthcare Workers Study, which was both initiated and led by frontline clinicians in partnership with academics. This study investigated the severity, prevalence and predictors of symptoms of mental illness, as well as the social, workplace and financial disruptions experienced by Australian HCWs during the COVID-19 pandemic.

\section{METHODS}

The second wave of the pandemic in Australia occurred predominantly in Melbourne, Victoria, between June and October 2020. Severe lockdown restrictions were instituted locally including (but not limited to) mandatory mask wearing; travel limited to $5 \mathrm{~km}$ from home; an evening curfew, 1-hour limit for outdoor exercise per day; limits on seeing extended family; working from home; home schooling; the closure of most shops, hospitality and entertainment venues; and closure of international and interstate borders.

\section{Participants and study design}

A nationwide, voluntary, anonymous, online survey was conducted between 27 August and 23 October 2020, concurrently with the second wave of the pandemic. Australian HCWs, comprising medical, nursing, allied health, medical laboratory, administrative and other support staff, who selfidentified as frontline HCWs in secondary or primary and community care, were invited to participate. Participants did not need to have cared for people with COVID-19 to participate. Over 8 weeks, 9518 survey responses were received,

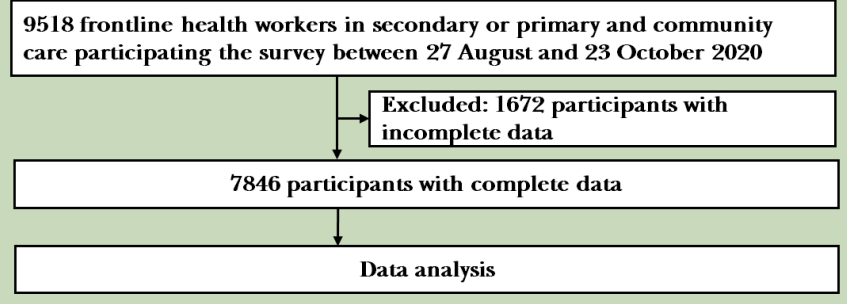

Figure 1 Flowchart of participant recruitment.

with complete data from $7846(82.4 \%)$ participants reported in this article (figure 1).

Participants were recruited through multiple strategies. Information regarding the survey was emailed to chief executive officers and departmental directors of frontline areas (emergency medicine, critical care, respiratory medicine, general medicine, infectious diseases, palliative care and hospital aged care) of all public hospitals throughout Victoria, and to multiple hospitals around Australia. Hospital leaders were asked to share the survey information with colleagues. Thirty-six professional societies, colleges, universities, associations and government health department staff also disseminated the information about the survey across Australia. In addition, the study was promoted through 117 newspapers, 8 television and radio news items and 30 social media sites.

\section{Data collection}

Each participant completed the survey once, with no longitudinal data collected. Participants completed the online survey either directly or via a purpose-built website (https://covid-19-frontline.com.au/). Before commencing the survey, participants provided online consent to participate. Data were collected and managed using REDCap electronic data capture tools. ${ }^{26}$

Information collected included demographics, home life details, professional background, work arrangements, the impact of the pandemic on employment and finances, organisational leadership, workplace change, exposure to COVID-19 and health and recreational habits (online supplemental file 1). Most questions were in a single-choice or multiple-choice format, with free text questions enabling more detailed answers. Five validated psychological measurement tools were completed to assess symptoms of mental illnesses: anxiety (Generalized Anxiety Disorder Scale-7 $($ GAD-7) $),{ }^{27}$ depression (Patient Health Questionnaire-9 (PHQ-9)), ${ }^{28}$ post-traumatic stress disorder (PTSD) (abbreviated Impact of Events Scale-6 (IES-6) $)^{29}$ and burnout (abbreviated Maslach Burnout Inventory (MBI) ${ }^{30}$ with subdomains of emotional exhaustion (EE), depersonalisation (DP) and personal accomplishment (PA)). Resilience was assessed using the abbreviated two-item Connor-Davidson Resilience Scale-2. ${ }^{31}$ Burnout on the MBI is indicated by higher scores on the EE and DP, and lower scores on the scale of PA. Cutoff scores for validated scales were as follows: depersonalisation: 0 to $3=$ low, 4 to $6=$ moderate, 7 to $18=$ high; emotional exhaustion: 0 to $6=$ low, 7 to $10=$ moderate, 11 to $18=$ high; 
personal accomplishment: 0 to $12=$ high, 13 to $14=$ moderate, 15 to $18=$ low ${ }^{32}$; IES is categorised as 0 to $9=$ none $/$ minimal and $>9=$ moderate-severe ${ }^{29} ; \mathrm{GAD}-7: 0$ to $4=$ none $/$ minimal, 5 to $9=$ mild, 10 to $14=$ moderate, 15 to $21=$ severe anxiety ${ }^{27}$; PHQ-9: 0 to $4=$ none $/$ minimal, 5 to $9=$ mild, 10 to $14=$ moderate, 15 to $19=$ moderately severe, 20 to $27=$ severe. ${ }^{28}$ In addition, participants were asked to report if they subjectively believed they had experienced anxiety, depression, PTSD, burnout or other mental health issues in order to determine their insight into their mental health. Ethics approval was provided by the Royal Melbourne Hospital Human Research Ethics Committee (HREC/67074/MH-2020).

\section{Statistical methods and data analysis}

A power calculation for general linear models was computed using RStudio. ${ }^{33}$ With an expected medium to large effect size and a power of 0.95 , and significance level of 0.05 , a sample of 6348 participants was required. To account for missing or incomplete data, a minimum sample size of 7000 responses was chosen. Data analysis was performed using SPSS V.26.0 statistical software (IBM). Demographic and socioeconomic characteristics were reported descriptively. Predictors of mental illness symptoms were identified through univariable logistic regression then entered into a multivariable logistic analyses included age; gender; state; occupation; number of working years since graduation; living situation (living alone, living with children, living with elderly); frontline area; practice location; working with patients with COVID19; anticipating working with patients with COVID-19; having received personal protective equipment (PPE) training; worry that their role will lead to COVID-19 transmission to family; worry regarding being blamed by colleagues, close friends or relatives infected with COVID-19; changed relationships with partner or friends or family or colleagues; changed household income; concerns regarding household income and pre-existing mental health diagnoses. For each mental illness scale, outcomes were merged into dichotomous categories (no or minimal symptoms vs moderate to severe symptoms) in the regression model. Associations between mental illness symptoms and predictor variables are presented as ORs with $95 \%$ CIs. Multicollinearity of predictor variables was examined using the variance inflation factor criterion. The Spearman coefficient $(r)$ was calculated to evaluate the correlation between self-reported and objective evidence of mental illness symptoms. For all statistical tests, significance was indicated by $\mathrm{p} \leq 0.05$.

\section{RESULTS}

\section{Demographic characteristics and workplace environment}

More than half $(n=4110,52.4 \%)$ of the participants were younger than 40 years, and $6344(80.9 \%)$ were women (table 1). Most participants were nurses $(\mathrm{n}=3088,39.4 \%)$, doctors $(\mathrm{n}=2436,31.0 \%)$ or allied health staff $(\mathrm{n}=1314$, $16.7 \%$ ) with 523 participants working in other health regression model. Covariates examined in univariable

Table 1 Participants' characteristics

\begin{tabular}{|c|c|c|}
\hline Characteristic & Frequency & $\%$ \\
\hline \multicolumn{3}{|l|}{ Age (years) $(n=7846)$} \\
\hline 20-30 & 1860 & 23.7 \\
\hline $31-40$ & 2250 & 28.7 \\
\hline $41-50$ & 1738 & 22.2 \\
\hline$>50$ & 1998 & 25.5 \\
\hline \multicolumn{3}{|l|}{ Gender $(n=7846)$} \\
\hline Male & 1458 & 18.6 \\
\hline Female & 6344 & 80.9 \\
\hline Non-binary & 19 & 0.2 \\
\hline Prefer not to say & 25 & 0.3 \\
\hline \multicolumn{3}{|l|}{ State $(n=7846)$} \\
\hline Victoria & 6685 & 85.2 \\
\hline New South Wales & 472 & 6.0 \\
\hline Queensland & 209 & 2.7 \\
\hline South Australia & 203 & 2.6 \\
\hline Western Australia & 137 & 1.7 \\
\hline Tasmania & 81 & 1.0 \\
\hline Australian Capital Territory & 35 & 0.4 \\
\hline Northern Territory & 24 & 0.3 \\
\hline \multicolumn{3}{|l|}{ Occupation (n=7846) } \\
\hline Nursing & 3088 & 39.4 \\
\hline Medical & 2436 & 31.0 \\
\hline Allied health & 1314 & 16.7 \\
\hline Administrative staff & 485 & 6.2 \\
\hline Other roles* & 523 & 6.7 \\
\hline \multicolumn{3}{|c|}{ Number of working years since graduated $(n=6637)$} \\
\hline $0-5$ & 1592 & 24.0 \\
\hline $6-10$ & 1377 & 20.7 \\
\hline $11-15$ & 943 & 14.2 \\
\hline$\geq 15$ & 2725 & 41.1 \\
\hline \multicolumn{3}{|c|}{ Number of people in the household $(n=7846)$} \\
\hline Living alone (1 person) & 1087 & 13.9 \\
\hline 2 & 2492 & 31.8 \\
\hline $3-4$ & 3181 & 40.5 \\
\hline $5-6$ & 1024 & 13.1 \\
\hline$\geq 7$ & 62 & 0.8 \\
\hline \multicolumn{3}{|c|}{ Number of children $<16$ years at home $(n=7846)$} \\
\hline 0 & 5102 & 65.0 \\
\hline $1-2$ & 2253 & 28.7 \\
\hline $3-4$ & 482 & 6.1 \\
\hline$\geq 5$ & 9 & 0.1 \\
\hline $\begin{array}{l}\text { Living with } \geq 1 \text { elderly person/people at } \\
\text { home }(n=7846)\end{array}$ & 697 & 8.9 \\
\hline \multicolumn{3}{|c|}{$\begin{array}{l}\text { *Other roles=pharmacists: } 185 \text {; clinical laboratory scientists } \\
\text { or technicians: } 176 \text {; paramedics: } 95 \text {; support staff (including } \\
\text { cleaning, security, facilities management personnel): } 43 \text {; } \\
\text { leadership role: } 9 \text {; other role: } 15 .\end{array}$} \\
\hline
\end{tabular}


organisation roles including food services and security. The medical staff group comprised 389 general practitioners, 1221 senior medical staff, 745 junior medical staff and 81 students. More than one-quarter of participants $(\mathrm{n}=2250,28.7 \%)$ had caring responsibilities at home, and $2133(27.2 \%)$ participants had children who were being homeschooled.

Participants worked in primary care or community roles $(n=1250,15.9 \%)$, medical specialty areas $(n=1205$, $15.4 \%)$, emergency departments $(\mathrm{n}=1146,14.6 \%)$, anaesthetics or surgical areas $(\mathrm{n}=824,10.5 \%)$ or intensive care units $(\mathrm{n}=745,9.5 \%)$ (table 2$)$. More than three-quarters $(\mathrm{n}=6158,78.5 \%)$ had been tested for COVID-19, 136 (1.7\%) had been infected with COVID-19 and 77 (0.9\%) had been previously quarantined because of unprotected exposure to COVID-19. Three-quarters $(\mathrm{n}=4551,76.4 \%)$ were worried or very worried that their role could lead to them transmitting COVID-19 to their families, and almost two-thirds $(\mathrm{n}=4949,63.1 \%)$ were worried about being blamed by colleagues for not taking adequate precautions if they contracted COVID-19.

\section{Relationship changes and prevalence of mental illness symptoms}

More than three-quarters of participants ( $\mathrm{n}=5994,76.4 \%)$ reported that the pandemic had affected their relationships with family, friends and colleagues, and nearly onethird had a close friend or relative who had been infected with COVID-19 either in Australia or overseas (table 2). Approximately one-third $(\mathrm{n}=2389,30.4 \%)$ reported having a pre-existing mental illness diagnosed before the pandemic (table 3). Many participants subjectively believed they had experienced mental illness during the pandemic including anxiety $(\mathrm{n}=4875,62.1 \%)$, burnout $(\mathrm{n}=4575,58.3 \%)$ and depression $(\mathrm{n}=2175,27.7 \%)$. Mental illness symptoms measured by objective scales demonstrated a similar or worse trend, with 4694 (59.8\%) participants experiencing mild to severe anxiety, 5458 (70.9\%) moderate to severe burnout (EE) and 4495 (57.3\%) mild to severe depression. Participants had a high score for resilience with a mean (SD) of $3.21(0.66)$ out of 4 . There was correlation between subjective reporting and objective evidence of moderate to severe mental illness symptoms for anxiety $(\mathrm{r}=0.346, \mathrm{p}<0.001)$, depression $(\mathrm{r}=0.346$, $\mathrm{p}<0.001)$ and $\mathrm{EE}(\mathrm{r}=0.354, \mathrm{p}<0.001)$.

\section{Predictors of poor mental health}

In the multivariable regression model, independent, personal predictors for worse mental health on all measured outcomes (anxiety, depression, burnout and PTSD) included female gender, younger age, experiencing worsening of personal relationships and low resilience scores (table 4). In addition, independent, personal predictors for anxiety and PTSD included having previous mental health conditions, having a family member or friend infected with COVID-19 and concerns about household income. Depression was also associated with having previous mental health conditions and concerns
Table 2 Work environment and relationship changes during the pandemic

\begin{tabular}{lrr}
\hline Characteristic & Frequency & $\%$ \\
\hline Frontline area ( $\mathrm{n}=7846)$ & & \\
\hline $\begin{array}{l}\text { Primary care or community } \\
\text { practitioner }\end{array}$ & 1250 & 15.9 \\
\hline Medical specialty areas & & \\
\hline Emergency department & 1205 & 15.4 \\
\hline Anaesthetics, perioperative care or & 1146 & 14.6 \\
\hline surgical areas & 824 & 10.5 \\
\hline Intensive care unit & 745 & 9.5 \\
\hline General medicine & 644 & 8.2 \\
\hline Hospital aged care & 536 & 6.8 \\
\hline Respiratory medicine & 336 & 4.3 \\
\hline Palliative care & 292 & 3.7 \\
\hline Infectious diseases & 171 & 2.2 \\
\hline Paramedicine & 99 & 1.3 \\
\hline Radiology & 61 & 0.8 \\
\hline Hospital pharmacy & 42 & 0.5 \\
\hline Pathology & 31 & 0.4 \\
\hline Worked in multiple or other areas $\dagger$ & 464 & 5.9 \\
\hline Location of practice $(\mathrm{n}=7846)$ & & \\
\hline Metropolitan & 6373 & 81.2 \\
\hline Regional & 1407 & 17.9 \\
\hline Remote & 66 & 0.8 \\
\hline
\end{tabular}

Currently working with people infected with COVID-19 $(n=7846)$

$\begin{array}{lll}\text { Yes } & 3063 & 39.0\end{array}$

$\begin{array}{lll}\text { No } & 4783 & 61.0\end{array}$

Number of patients infected with $1.4(0.43)$

COVID-19 cared for, mean (SD)

Anticipating working with people infected with COVID-19 $(n=4775)$

$\begin{array}{lll}\text { Yes } & 2891 & 60.5 \\ \text { No } & 1884 & 39.5\end{array}$

Received training on PPE during the pandemic $(n=7846)$

$\begin{array}{lll}\text { Yes } & 5137 & 65.5 \\ \text { No } & 2709 & 34.5\end{array}$

Being worried that their roles will lead to them transmitting COVID-19 to family $(n=5954)$

$\begin{array}{lrr}\text { Not worried } & 729 & 12.2 \\ \text { Neutral } & 674 & 11.3 \\ \text { Worried or very worried } & 4551 & 76.4\end{array}$

Being worried about being blamed by colleagues if they contract COVID-19 $(n=7846)$

$\begin{array}{lcc}\text { Neutral } & 1275 & 16.3 \\ \text { Not worried } & 1622 & 20.7 \\ \text { Worried } & 4949 & 63.1\end{array}$

Experiencing close friends/relatives infected with COVID-19 in Australia or overseas $(n=7846)$

Yes

2398 30.6 Continued 
Table 2 Continued

\begin{tabular}{|c|c|c|}
\hline Characteristic & Frequency & $\%$ \\
\hline No & 5448 & 69.4 \\
\hline \multicolumn{3}{|c|}{ Impact of COVID-19 on relationships $(n=7846)$} \\
\hline \multicolumn{3}{|c|}{ Closer or stronger relationship with } \\
\hline Partner & 2266 & 28.9 \\
\hline Children/parents/family & 2226 & 28.4 \\
\hline Friends & 1054 & 13.4 \\
\hline Work colleagues & 2533 & 32.3 \\
\hline \multicolumn{3}{|l|}{ Worse relationship with } \\
\hline Partner & 1000 & 12.7 \\
\hline Children/parents/family & 1421 & 18.1 \\
\hline Friends & 2221 & 28.3 \\
\hline Work colleagues & 1116 & 14.2 \\
\hline No effect on relationships & 1852 & 23.6 \\
\hline \multicolumn{3}{|c|}{ Change in household income due to COVID-19 $(n=7846)$} \\
\hline Increased & 820 & 10.5 \\
\hline Decreased & 2415 & 30.8 \\
\hline No change & 4611 & 58.8 \\
\hline
\end{tabular}

Concerns or worries about household income since COVID-19 $(\mathrm{n}=7846)$

$\begin{array}{lll}\text { Yes } & 2416 & 30.8 \\ \text { No } & 5430 & 69.2\end{array}$

*Medical specialty areas included all medical specialties other than hospital aged care, general medicine, respiratory medicine, palliative care and infectious diseases. The latter were reported separately due to their potentially increased risk of exposure to COVID-19.

†This group included (but was not limited to) people working in leadership roles, clerical roles, support roles, food preparation, facilities maintenance, screening clinics and clinical scientists. COVID-19, coronavirus disease 2019; PPE, personal protective equipment.

about household income, whereas EE was also associated with previous mental health conditions. Independent, workplace predictors for worse mental health outcomes on all measured scales (anxiety, depression, burnout and PTSD) included having a nursing, allied health or other non-medical role, frontline area, working with patients infected with COVID-19 and being worried about being blamed by colleagues on contracting COVID-19 infection (table 5). There were no significant associations between other demographic, work environment, relationship or financial covariates and each mental illness score.

\section{DISCUSSION}

\section{Main findings}

To our knowledge, this is the largest, national, crosssectional study examining psychosocial distress during the COVID-19 pandemic in Australia that has included all frontline healthcare occupations and areas. Despite participants receiving high scores on the validated
Table 3 Prevalence of mental health issues

\begin{tabular}{|c|c|c|}
\hline Characteristic & Frequency & $\%$ \\
\hline \multicolumn{3}{|c|}{ Pre-existing mental health condition diagnosed before the pandemic $(n=7846)$} \\
\hline Yes & 2389 & 30.4 \\
\hline No & 5272 & 67.2 \\
\hline Prefer not to say & 185 & 2.4 \\
\hline \multicolumn{3}{|c|}{ Self-reported mental health issues experienced since COVID-19 $(n=7846)^{*}$} \\
\hline Anxiety & 4875 & 62.1 \\
\hline Burnout & 4575 & 58.3 \\
\hline Depression & 2175 & 27.7 \\
\hline PTSD & 427 & 5.4 \\
\hline Other mental health issues & 328 & 4.2 \\
\hline No mental health issues & 1431 & 18.2 \\
\hline \multicolumn{3}{|c|}{ Mental health issues assessed by validated scales } \\
\hline \multicolumn{3}{|l|}{ Burnout DP $(\mathrm{n}=7688)$} \\
\hline Low & 4811 & 62.6 \\
\hline Moderate & 1321 & 17.2 \\
\hline High & 1556 & 20.2 \\
\hline \multicolumn{3}{|l|}{ Burnout $E E(\mathrm{n}=7701)$} \\
\hline Low & 2243 & 29.1 \\
\hline Moderate & 2079 & 27.0 \\
\hline High & 3379 & 43.9 \\
\hline \multicolumn{3}{|l|}{ Burnout PA $(\mathrm{n}=7689)$} \\
\hline Low & 2358 & 30.7 \\
\hline Moderate & 1592 & 20.7 \\
\hline High & 3739 & 48.6 \\
\hline \multicolumn{3}{|l|}{ Anxiety $-G A D-7(\mathrm{n}=7843)$} \\
\hline None/minimal & 3149 & 40.2 \\
\hline Mild & 2478 & 31.6 \\
\hline Moderate & 1293 & 16.5 \\
\hline Severe & 923 & 11.8 \\
\hline \multicolumn{3}{|l|}{ Depression-PHQ-9 $(\mathrm{n}=7841)$} \\
\hline None/minimal & 3321 & 42.5 \\
\hline Mild & 2303 & 29.5 \\
\hline Moderate & 1203 & 15.4 \\
\hline Moderately severe & 620 & 7.9 \\
\hline Severe & 369 & 4.7 \\
\hline \multicolumn{3}{|c|}{ Impact of events/trauma-IES-6 $(n=7796)$} \\
\hline None/minimal & 4641 & 59.5 \\
\hline \multirow{2}{*}{ Moderate-severe } & 3155 & 40.5 \\
\hline & Mean (SD) & Range \\
\hline Resilience-CD-RISC2 ( $n=7841)$ & $3.21(0.66)$ & $0-4$ \\
\hline
\end{tabular}

Burnout DP: 0 to $3=$ low, 4 to $6=$ moderate, 7 to $18=$ high. Burnout EE: 0 to $6=$ low, 7 to $10=$ moderate, 11 to $18=$ high. Burnout PA: 0 to $12=$ high burnout, 13 to $14=$ moderate burnout, 15 to $18=$ low burnout. IES is categorised as 0to $9=$ none/ minimal and $>9=$ moderate-severe. GAD-7: 0 to $4=$ none/minimal, 5 to $9=$ mild, 10 to $14=$ moderate, 15 to $21=$ severe anxiety. PHQ-9: 0 to $4=$ none/minimal, 5 to $9=$ mild, 10 to $14=$ moderate, 15 to $19=$ =moderately severe, 20 to $27=$ severe. *Multiple options could be chosen.

CD-RISC2, Connor-Davidson Resilience Scale-2; COVID-19, coronavirus disease 2019; DP, depersonalisation; EE, emotional exhaustion; GAD-7, Generalized Anxiety Disorder Scale-7; IES-6, Impact of Events Scale-6; PA, personal accomplishment; PHQ-9, Patient Health Questionnaire-9; PTSD, post-traumatic stress disorder.

resilience instrument, the majority experienced anxiety or depressive symptoms, or EE (burnout). This indicates that the protective effects of resilience are not sufficient 


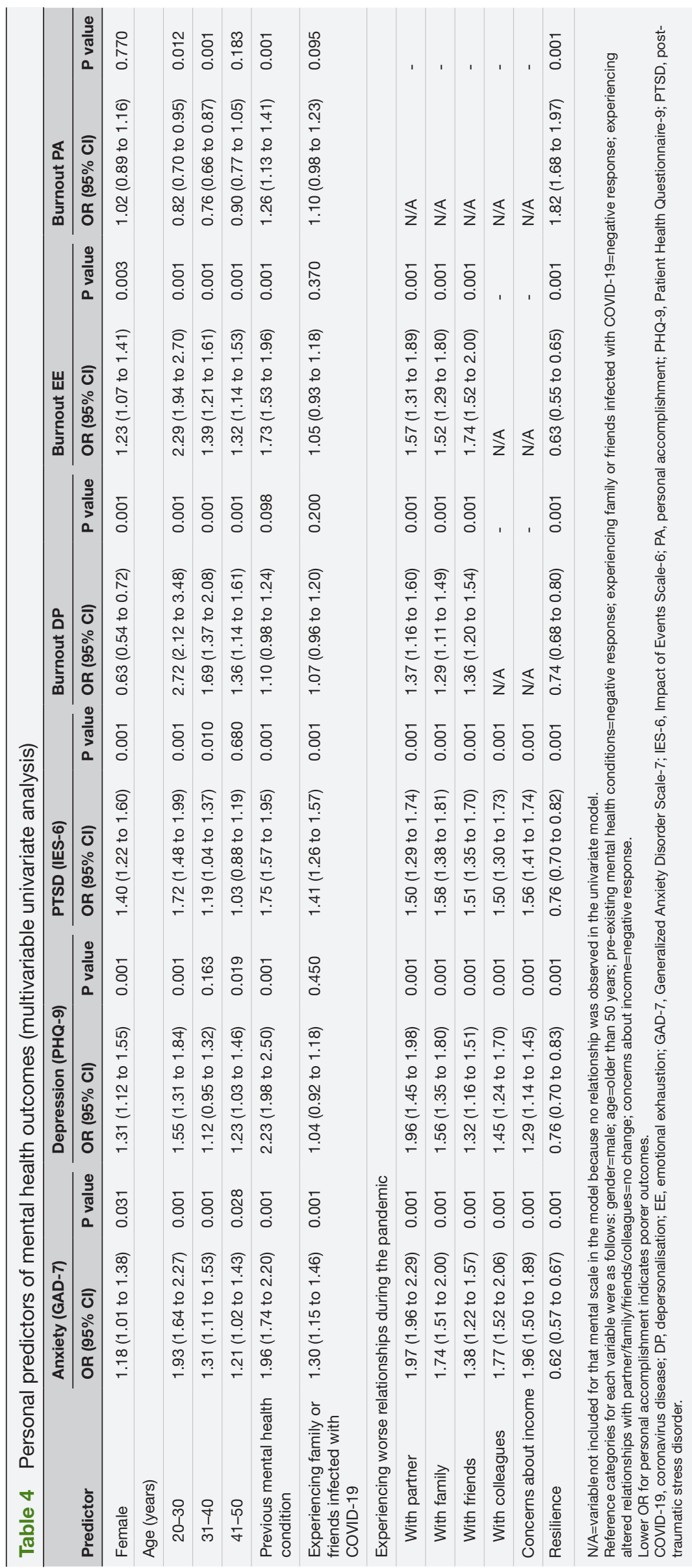




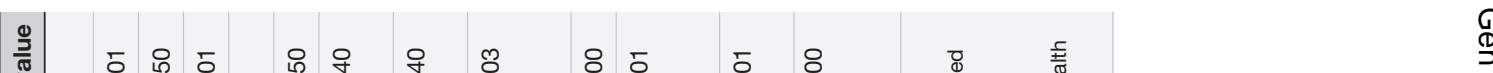

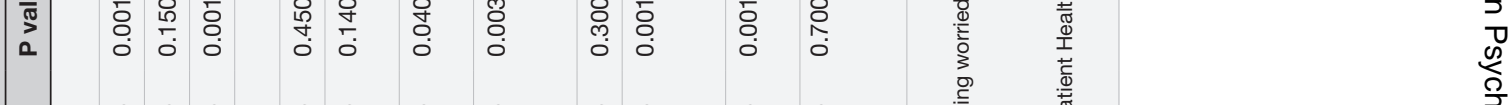

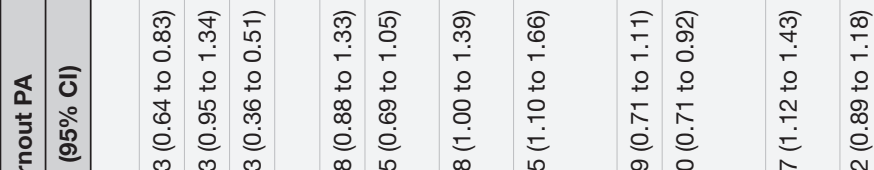

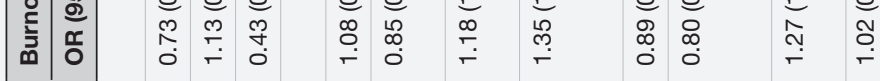

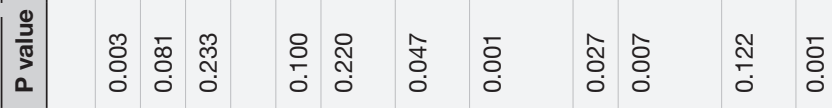

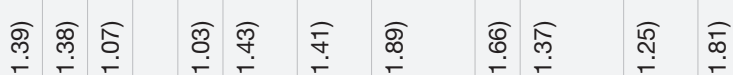

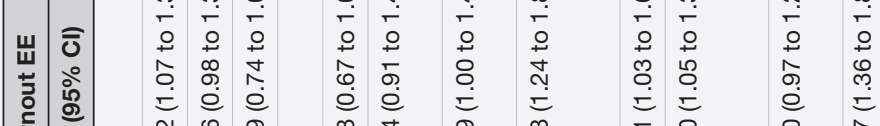

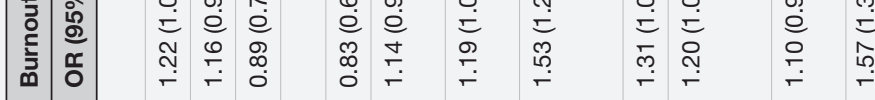

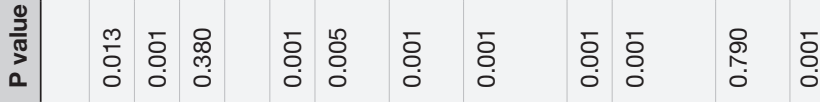

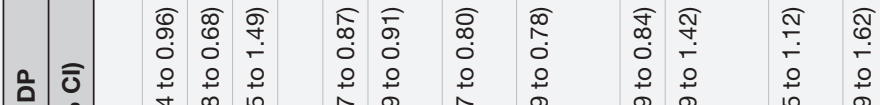

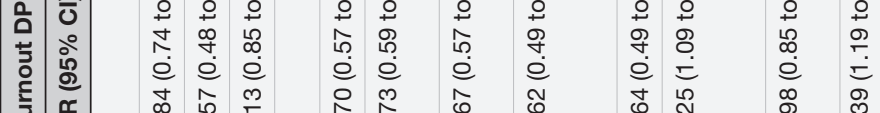

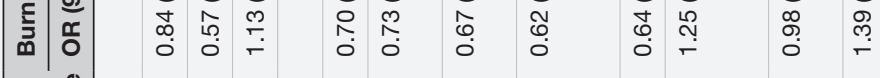

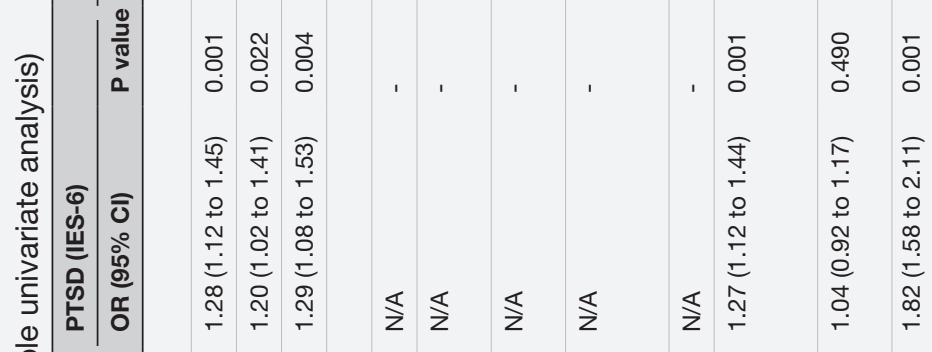

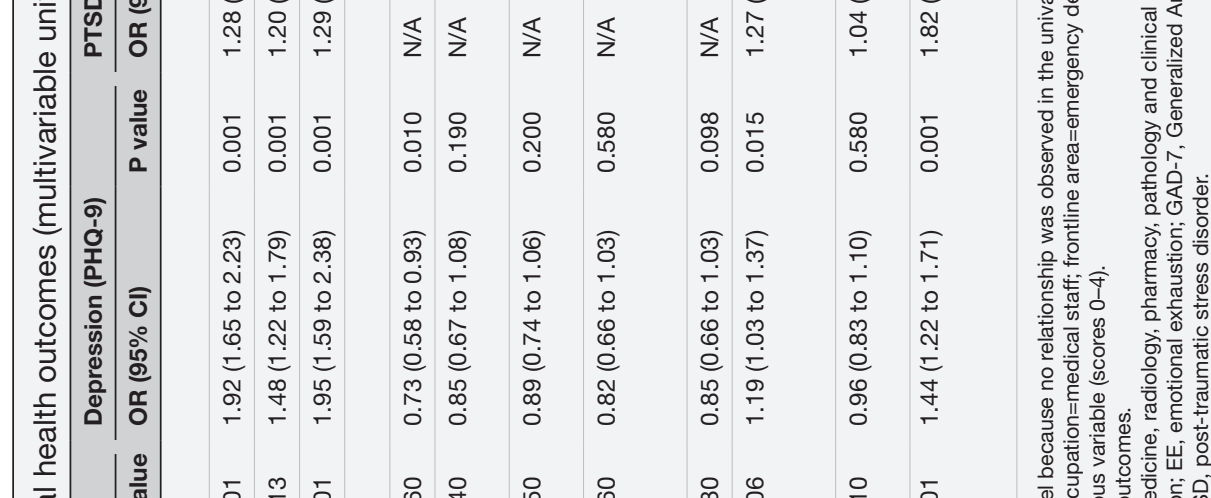

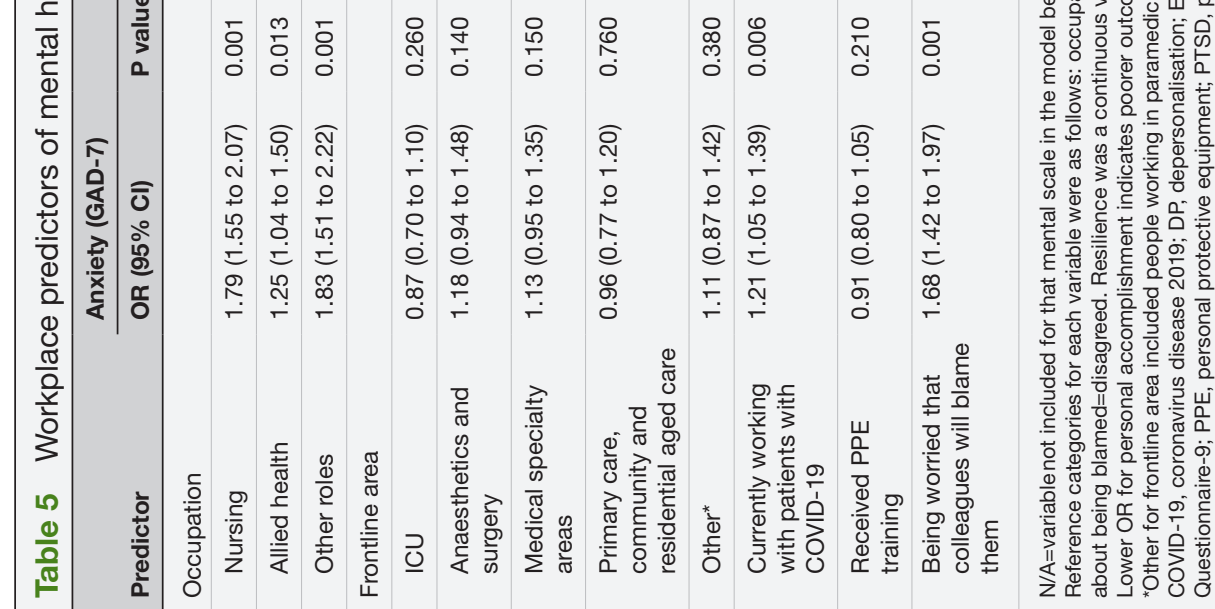


to prevent psychological harm during the pandemic. A significant proportion also experienced PTSD symptoms. Although less than half of the participants worked with patients with COVID-19 and very few had been infected with COVID-19 or quarantined, many experienced disruptions to family life, altered social relationships and financial worries. Our findings are consistent with those reported in international studies: high mental health burden on frontline workers during COVID-19 101115 and SARS pandemics. ${ }^{34}$ Fears of transmitting COVID-19 infection to family and of being blamed by colleagues for not taking adequate precautions if they did contract COVID-19 were extremely common. Personal, social and workplace predictors for mental illness symptoms have been identified.

Around the world, a growing number of largely country-specific, single timepoint, cross-sectional surveys have identified that mental health problems are common in HCWs during the COVID-19 pandemic. Prevalence estimates are as follows: $33 \%$ to $59 \%$ for anxiety, $30 \%$ to $62 \%$ for depression, $41 \%$ to $51 \%$ for burnout and approximately $57 \%$ for acute distress or PTSD. ${ }^{10}{ }^{11} 13-1535$ The upper limits of these prevalence estimates are strikingly similar to our own findings. However, moderate to severe burnout (EE) was much more prevalent in our study $(70.9 \%)$, which may be explained by the later timing of our study, by which time Australian HCWs had endured many months of social and workplace disruptions, and lockdown restrictions.

By contrast, two separate, small $(n=320$ and $n=668)$, single-site, single timepoint surveys of HCWs undertaken in Melbourne from April to May 2020 and from May to June 2020 both identified a lower prevalence of adverse mental health outcomes. ${ }^{36}{ }^{37}$ Their findings may again be partly explained by the earlier timing of the studies in the first wave and the lack of power in those studies due to smaller size of the samples. Comparing our data to international data, the high prevalence of symptoms of poor mental health in our study is interesting given the comparatively low case load of COVID-19 in Australia. One explanation is that anticipation and fear of a catastrophic crisis leading to high death rates of patients and HCWs (as Australian HCWs saw occurring overseas) contributed to adverse psychological outcomes. ${ }^{14}$ This concept of psychological distress being related to anticipated, perceived risk is important and highlights the critical importance of crisis preparedness, good government and organisational leadership and consistent clear communication. In addition, the pervasive media coverage regarding COVID-19 along with the many restrictions enacted in local lockdowns may have contributed to poor mental health in Australian frontline workers.

Similar to our findings, studies from overseas have found that predictors of poor mental health in HCWs during the pandemic include female gender, less years of work experience (which in our study correlated with younger age), pre-existing psychological illnesses, working in a nursing role and working in certain frontline areas. ${ }^{10-13} 1516353839$
Many of these groups are at heightened risk of psychosocial harm during non-pandemic times, and it is possible that crises such as COVID-19 exacerbate harm in preexisting vulnerable groups. ${ }^{40}$ Importantly, unlike previous small local and international studies, the large sample size in our study enabled us to demonstrate that female gender and working in nursing or allied health roles are independent predictors of poor mental health. The relationship between nursing and poorer mental health may be explained by the heightened risk of COVID-19 exposure from prolonged and frequent contact with patients. Moreover, nursing and allied health professionals generally have less choice regarding their daily work environments. ${ }^{11-1316}$ Reduced finances were not associated with a nursing role and therefore did not explain the association.

The relationship between gender and adverse mental illness outcomes is intriguing, given that this relationship was identified even during the SARS pandemic. ${ }^{34}$ One possible explanation is that men and women have different coping styles, ${ }^{41}$ with men having greater odds of reporting DP in this study. In addition, a British study identified that women have had to bear greater responsibilities (on average, an extra 11.2 hours of unpaid work per week) than men as primary carers for dependents during the pandemic. ${ }^{42}$ General population data from the Australian Bureau of Statistics report similar findings, with women three times more likely than men to perform the majority of caregiving tasks and twice as likely to undertake the majority of unpaid domestic work. ${ }^{43}$ In our study, having young or old dependents was not a predictor of poor mental health. However, we did not specifically enquire about the number of additional unpaid hours undertaken in the home for domestic or caregiving tasks during the pandemic. As there was no difference in resilience scores between men and women, this gender difference requires further exploration. The lack of a relationship between PPE training and poor mental health in our study may relate to the majority of frontline staff receiving training and the relatively low rates of COVID-19 infection in Australia compared with other countries.

\section{Limitations}

The large sample size in our study enabled detailed examination of independent predictors of poor mental health. Most participants in our study were women, which is consistent with data from both the Australian Institute of Health and Welfare and the Australian Health Practitioner Regulation Agency demonstrating that $75 \%$ of the Australian health workforce is female. ${ }^{44-46}$ Because of the very broad survey dissemination strategy, calculation of a response rate was not possible. Selection bias and response bias may have led to overestimation or underestimation of psychological distress and rates of pre-existing mental health illness. Similarly, in line with other international surveys exploring the psychosocial effects of the COVID-19 pandemic on healthcare workers, we were not able to confirm clinical diagnoses of mental illness with 
the symptoms measured by the validated psychological scales. Nevertheless, these scales are validated and the only feasible option for measuring mental health symptoms in a large-scale survey such as this.

Because of the spontaneous and unexpected nature of the COVID-19 pandemic, no baseline data regarding mental health symptoms in non-pandemic times had previously been collected from a large cohort of Australian HCWs. Therefore, it is not possible to demonstrate a change in the prevalence estimates of mental health symptoms in this study. Nevertheless, the prevalence estimates in this study are much higher than those reported in earlier studies in non-pandemic times. ${ }^{247-49}$ Notably, the case load of COVID-19 in Australia at the time of survey closure was low relative to international settings, with 27484 cases recorded. ${ }^{50}$ The prevalence of mental health impacts arising in the Australian context is indicative of harm related to the prolonged stress of a pandemic, even with relatively few cases. Participant responses were measured at a single timepoint, not longitudinally, to avoid excessively burdening the frontline healthcare workers during the pandemic. However, given the ongoing nature of the pandemic, we believe that longitudinal research is urgently required to better understand any persisting psychosocial effects of the pandemic on HCWs and any ramifications for patient safety and workforce retention. Similar prospective studies sampling Italian HCWs during the first and second waves of COVID-19 have reported growing prevalence of mental health issues as the pandemic continues, and it is likely that similar trends exist in Australia. ${ }^{51}$ Furthermore, research is required to examine the acceptability, uptake and effectiveness of any new interventions introduced to support the well-being of HCWs.

\section{Implications}

Although many factors, including lockdown restrictions, social disconnection and media coverage, likely have contributed to the high prevalence of mental health symptoms in frontline healthcare workers in this study, occupational factors cannot be ignored. Indeed, occupational factors (related to workloads, training, PPE, organisational leadership, communication and policies) must be actively considered because they represent important opportunities to intervene and prevent mental health issues. Both better crisis preparedness and new psychological support services for HCWs are needed. Importantly, such services should not just be short-term 'fixes' to address the current pandemic-related issues, but instead should provide long-term support given the high prevalence of pre-existing mental health diagnoses. These supports must be accessible and acceptable to HCWs. Although resilience was identified as a protective factor in this study, the overall resilience level of HCWs was already high, and as such, approaches that aim to build resilience are likely to have limited efficacy in this cohort. Furthermore, it is vital that health leaders in the government, secondary care and the community recognise that certain groups of HCWs are more vulnerable to mental health problems and therefore require additional targeted support interventions. Crucially important are organisational policies and practices that address burnout (and contributing factors such as information overload), given its extremely high prevalence and the risk it poses to workforce retention. ${ }^{7}$

The health workforce is an indispensable asset. Yet crises such as the COVID-19 pandemic are associated with significant mental health symptoms in frontline HCWs, with potentially wide repercussions for individuals, patients and the workforce. Crisis preparedness, along with long-term, evidence-based policies and practices that focus on preventing and actively addressing psychological well-being, is needed to protect, maintain and 'futureproof' the health workforce.

\section{Author affiliations}

${ }^{1}$ Department of Respiratory Medicine, Alfred Hospital, Prahran, Victoria, Australia

${ }^{2}$ Department of Allergy, Immunology and Respiratory Medicine, Central Clinical

School, Alfred Hospital, Monash University, Melbourne, Victoria, Australia

${ }^{3}$ School of Psychology and Public Health, La Trobe University, Melbourne, Victoria, Australia

${ }^{4}$ School of Medicine and Healthcare Management, Caucasus University, Tbilisi, Georgia

${ }^{5}$ Department of Psychiatry, The Royal Melbourne Hospital, Parkville, Victoria, Australia

${ }^{6}$ Department of Public Health Law, Melbourne School of Population and Global Health, The University of Melbourne, Parkville, Victoria, Australia

${ }^{7}$ Department of Emergency Services, The Royal Melbourne Hospital, Parkville, Victoria, Australia

${ }^{8}$ Department of Critical Care, Faculty of Medicine, Dentistry and Health Sciences, The University of Melbourne, Melbourne, Victoria, Australia

${ }^{9}$ Departments of General Medicine and Infectious Diseases, The Royal Melbourne Hospital, Parkville, Victoria, Australia

${ }^{10}$ Department of Medicine, The Royal Melbourne Hospital, The University of Melbourne, Parkville, Victoria, Australia

${ }^{11}$ Allergy and Lung Health Unit, School of Population and Global Health, The University of Melbourne, Parkville, Victoria, Australia

${ }^{12}$ Department of Allied Health, The Royal Melbourne Hospital, Parkville, Victoria, Australia

${ }^{13}$ Parkville Integrated Palliative Care Service, The Royal Melbourne Hospital, Parkville, Victoria, Australia

${ }^{14}$ Sir Peter MacCallum Department of Oncology, The University of Melbourne, Parkville, Victoria, Australia

${ }^{15}$ Department of Geriatric Medicine, Western Health, Footscray, Victoria, Australia

${ }^{16}$ Department of Anaesthesia and Pain Management, The Royal Melbourne Hospital,

Parkville, Victoria, Australia

${ }^{17}$ Centre for Integrated Critical Care, Melbourne Medical School, The University of Melbourne, Parkville, Victoria, Australia

${ }^{18}$ Department of Physiotherapy, Alfred Health, Melbourne, Victoria, Australia

${ }^{19}$ Rheumatology Unit, Royal Children's Hospital, Parkville, Victoria, Australia

${ }^{20}$ Arthritis and Rheumatology, Murdoch Children's Research Institute, Parkville, Victoria, Australia

${ }^{21}$ Department of Infectious Diseases, The Royal Melbourne Hospital, Parkville, Victoria, Australia

${ }^{22}$ Department of Intensive Care Medicine, The Royal Melbourne Hospital, Parkville, Victoria, Australia

${ }^{23}$ School of Nursing and Midwifery, College of Science, Health and Engineering, La Trobe University, Melbourne, Victoria, Australia

${ }^{24}$ Royal Adelaide Hospital, University of South Australia, Adelaide, South Australia, Australia

${ }^{25}$ College of Health and Biomedicine, Victoria University, Footscray, Victoria, Australia ${ }^{26}$ Division of Critical Care and Investigative Services, The Royal Melbourne Hospital, Parkville, Victoria, Australia 
Acknowledgements We gratefully acknowledge and thank The Royal Melbourne Hospital Foundation and the Lord Mayor's Charitable Foundation for financial support for this study. We wish to thank the numerous health organisations, universities, professional societies, associations and colleges, and many supportive individuals who assisted in disseminating the survey. We thank The Royal Melbourne Hospital Business Intelligence Unit who provided and hosted the REDCap electronic data capture tools.

Contributors NS, KW: conceptualisation, data curation, formal analysis, funding acquisition, investigation, methodology, project administration, resources, supervision, writing (original and revisions). LK: conceptualisation, formal analysis, investigation, methodology, resources, supervision, writing (original and revisions). MB, MP, DJ, SCD, EB, NA, CL, IN, AH, JEM, IT, CM, AM, DS: conceptualisation, investigation, methodology, writing (original).

Funding The Royal Melbourne Hospital Foundation and the Lord Mayor's Charitable Foundation kindly provided financial support for this study.

Disclaimer Funding bodies had no role in the research activity. All authors were independent of the funders and had access to the study data.

Competing interests None declared.

Patient consent for publication Not required.

Provenance and peer review Not commissioned; externally peer reviewed.

Data availability statement Data are available upon reasonable request. Data are available upon reasonable request from the corresponding author.

Supplemental material This content has been supplied by the author(s). It has not been vetted by BMJ Publishing Group Limited (BMJ) and may not have been peer-reviewed. Any opinions or recommendations discussed are solely those of the author(s) and are not endorsed by BMJ. BMJ disclaims all liability and responsibility arising from any reliance placed on the content. Where the content includes any translated material, BMJ does not warrant the accuracy and reliability of the translations (including but not limited to local regulations, clinical guidelines, terminology, drug names and drug dosages), and is not responsible for any error and/or omissions arising from translation and adaptation or otherwise.

Open access This is an open access article distributed in accordance with the Creative Commons Attribution Non Commercial (CC BY-NC 4.0) license, which permits others to distribute, remix, adapt, build upon this work non-commercially, and license their derivative works on different terms, provided the original work is properly cited, appropriate credit is given, any changes made indicated, and the use is non-commercial. See: http://creativecommons.org/licenses/by-nc/4.0/.

ORCID iD

Mark Putland http://orcid.org/0000-0002-1994-252X

\section{REFERENCES}

1 De Cieri H, Shea T, Cooper B, et al. Effects of work-related stressors and mindfulness on mental and physical health among Australian nurses and healthcare workers. J Nurs Scholarsh 2019;51:580-9.

2 Imo UO. Burnout and psychiatric morbidity among doctors in the UK: a systematic literature review of prevalence and associated factors. BJPsych Bull 2017;41:197-204.

3 Milner AJ, Maheen H, Bismark MM, et al. Suicide by health professionals: a retrospective mortality study in Australia, 2001-2012. Med J Aust 2016;205:260-5.

4 Petrie K, Crawford J, Baker STE, et al. Interventions to reduce symptoms of common mental disorders and suicidal ideation in physicians: a systematic review and meta-analysis. Lancet Psychiatry 2019;6:225-34.

5 Courtney JA, Francis AJP, Paxton SJ. Caring for the country: fatigue, sleep and mental health in Australian rural paramedic shiftworkers. $J$ Community Health 2013;38:178-86.

6 Tawfik DS, Scheid A, Profit J, et al. Evidence relating health care provider burnout and quality of care: a systematic review and metaanalysis. Ann Intern Med 2019;171:555-67.

7 Salyers MP, Flanagan ME, Firmin R, et al. Clinicians' perceptions of how burnout affects their work. Psychiatr Serv 2015;66:204-7.

8 Bai Y, Lin C-C, Lin C-Y, et al. Survey of stress reactions among health care workers involved with the SARS outbreak. Psychiatr Serv 2004;55:1055-7.

9 Lee AM, Wong JGWS, McAlonan GM, et al. Stress and psychological distress among SARS survivors 1 year after the outbreak. Can J Psychiatry 2007;52:233-40.
10 Azoulay E, De Waele J, Ferrer R, et al. Symptoms of burnout in intensive care unit specialists facing the COVID-19 outbreak. Ann Intensive Care 2020;10:110.

11 Luceño-Moreno L, Talavera-Velasco B, García-Albuerne Y, et al. Symptoms of posttraumatic stress, anxiety, depression, levels of resilience and burnout in Spanish health personnel during the COVID-19 pandemic. Int J Environ Res Public Health 2020;17:30.

12 Matsuo T, Kobayashi D, Taki F, et al. Prevalence of health care worker burnout during the coronavirus disease 2019 (COVID-19) pandemic in Japan. JAMA Netw Open 2020;3:e2017271-e:e2017271.

13 Shechter A, Diaz F, Moise N, et al. Psychological distress, coping behaviors, and preferences for support among New York healthcare workers during the COVID-19 pandemic. Gen Hosp Psychiatry 2020;66:1-8.

14 Temsah M-H, Al-Sohime F, Alamro N, et al. The psychological impact of COVID-19 pandemic on health care workers in a MERS-CoV endemic country. J Infect Public Health 2020;13:877-82.

15 WZY T, Soo YE, Yip C. The psychological impact of COVID-19 on 'hidden' frontline healthcare workers. Int J Soc Psychiatry 2021;67.

16 Zhu Z, Xu S, Wang H, et al. COVID-19 in Wuhan: sociodemographic characteristics and hospital support measures associated with the immediate psychological impact on healthcare workers. EClinicalMedicine 2020;24:100443.

17 Oates J, Drey N, Jones J. Associations between age, years in post, years in the profession and personal experience of mental health problems in UK mental health nurses. Issues Ment Health Nurs 2017;38:624-32.

18 Hayes B, Prihodova L, Walsh G, et al. What's up doc? A national cross-sectional study of psychological wellbeing of hospital doctors in Ireland. BMJ Open 2017;7:e018023.

19 Petrie K, Crawford J, LaMontagne AD, et al. Working hours, common mental disorder and suicidal ideation among junior doctors in Australia: a cross-sectional survey. BMJ Open 2020;10:e033525.

20 Pyper Z, Paterson JL. Fatigue and mental health in Australian rural and regional ambulance personnel. Emerg Med Australas 2016;28:62-6.

21 Colville GA, Smith JG, Brierley J, et al. Coping with staff burnout and work-related posttraumatic stress in intensive care. Pediatr Crit Care Med 2017;18:e267-73.

22 Bu F, Steptoe A, Fancourt D. Loneliness during lockdown: trajectories and predictors during the COVID-19 pandemic in 35,712 adults in the UK. medRxiv 2020

23 Bu F, Steptoe A, Fancourt D. Who is lonely in lockdown? Crosscohort analyses of predictors of loneliness before and during the COVID-19 pandemic. medRxiv 2020

24 Garnett C, Jackson S, Oldham M, et al. Factors associated with drinking behaviour during COVID-19 social distancing and lockdown among adults in the UK. Drug Alcohol Depend 2021;219:108461.

25 lob E, Steptoe A, Fancourt D, Abuse FD. Abuse, self-harm and suicidal ideation in the UK during the COVID-19 pandemic. $\mathrm{Br} J$ Psychiatry 2020;217:543-6.

26 Harris PA, Taylor R, Thielke R, et al. Research electronic data capture (REDCap)--a metadata-driven methodology and workflow process for providing translational research informatics support. J Biomed Inform 2009;42:377-81.

27 Spitzer RL, Kroenke K, Williams JBW, et al. A brief measure for assessing generalized anxiety disorder: the GAD-7. Arch Intern Med 2006;166:1092-7.

28 Kroenke K, Spitzer RL, Williams JB. The PHQ-9: validity of a brie depression severity measure. J Gen Intern Med 2001;16:606-13.

29 Thoresen S, Tambs K, Hussain A, et al. Brief measure of posttraumatic stress reactions: Impact of Event Scale-6. Soc Psychiatry Psychiatr Epidemiol 2010;45:405-12.

30 Maslach C, Jackson SE, Leiter MP. Maslach burnout inventory: third edition. evaluating stress: a book of resources. Lanham, MD, US: Scarecrow Education, 1997: 191-218.

31 Vaishnavi S, Connor K, Davidson JRT. An abbreviated version of the Connor-Davidson Resilience Scale (CD-RISC), the CD-RISC2: psychometric properties and applications in psychopharmacological trials. Psychiatry Res 2007;152:293-7.

32 Riley MR, Mohr DC, Waddimba AC. The reliability and validity of three-item screening measures for burnout: evidence from groupemployed health care practitioners in upstate New York. Stress Health 2018;34:187-93.

33 RStudio Team. RStudio: integrated development environment for $R$ MA: Boston, 2015. http://www.rstudio.com/

34 Tam CWC, Pang EPF, Lam LCW, et al. Severe acute respiratory syndrome (SARS) in Hong Kong in 2003: stress and psychological impact among frontline healthcare workers. Psychol Med 2004;34:1197. 
35 Hu D, Kong Y, Li W, et al. Frontline nurses' burnout, anxiety, depression, and fear statuses and their associated factors during the COVID-19 outbreak in Wuhan, China: a large-scale cross-sectional study. EClinicalMedicine 2020;24:100424.

36 Dobson $\mathrm{H}$, Malpas CB, Burrell AJ, et al. Burnout and psychological distress amongst Australian healthcare workers during the COVID-19 pandemic. Australas Psychiatry 2021;29:26-30.

37 Holton S, Wynter K, Trueman M, et al. Psychological well-being of Australian hospital clinical staff during the COVID-19 pandemic. Aust Health Rev 2020.

38 Arpacioglu S, Gurler M, Cakiroglu S. Secondary traumatization outcomes and associated factors among the health care workers exposed to the COVID-19. Int J Soc Psychiatry 2021;67:84-9.

39 Zhang W-R, Wang K, Yin L, et al. Mental health and psychosocial problems of medical health workers during the COVID-19 epidemic in China. Psychother Psychosom 2020;89:242-50.

40 Magnavita N, Chirico F, Garbarino S, et al. SARS/MERS/SARSCoV-2 outbreaks and burnout syndrome among healthcare workers. An umbrella systematic review. Int $J$ Environ Res Public Health 2021;18:4361.

41 Lebares CC, Braun HJ, Guvva EV, et al. Burnout and gender in surgical training: a call to re-evaluate coping and dysfunction. $A m \mathrm{~J}$ Surg 2018;216:800-4.

42 Strauss P-C. COVID-19 and the female health and care workforce - Survey of health and care staff for the Health and Care Women Leaders Network, 2020.

43 Australian Bureau of Statistics. Household impacts of COVID-19 survey: coronavirus (COVID-19) impacts on jobs, unpaid care, domestic work, mental health and related services, and life after COVID-19 restrictions, 2020. Available: https://www.abs.gov.au/ statistics/people/people-and-communities/household-impactscovid-19-survey/6-10-july-2020

44 Australian Institute of Health and Welfare. Health workforce. Canberra: Australian Institute of Health and Welfare, 2020. https:// www.aihw.gov.au/reports/australias-health/health-workforce

45 Medical Board of Australia. Registration Data Table - December 2020 2020 10th February, 2021.

46 Nursing and Midwifery Board. Nursing and midwifery board of Australia Registrant data, 2020.

47 Hollingsworth CE, Wesley C, Huckridge J, et al. Impact of child death on paediatric trainees. Arch Dis Child 2018;103:14-18.

48 Thompson CV, Naumann DN, Fellows JL, et al. Post-traumatic stress disorder amongst surgical trainees: an unrecognised risk? Surgeon 2017:15:123-30.

49 Leinweber J, Creedy DK, Rowe H, et al. Responses to birth trauma and prevalence of posttraumatic stress among Australian midwives. Women Birth 2017;30:40-5.

50 Australian Government Department of Health. Coronavirus (COVID-19) at a glance - 23 October 2020, 2020. Available: https:// www.health.gov.au/resources/publications/coronavirus-covid-19-ata-glance-23-october-2020

51 Magnavita N, Soave PM, Antonelli M. Prolonged stress causes depression in frontline workers facing the COVID-19 pandemic-A repeated cross-sectional study in a COVID-19 hub-hospital in centra Italy. Int J Environ Res Public Health 2021;18:7316.

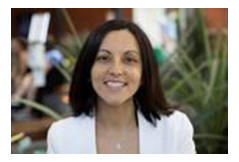

Associate Professor Natasha Smallwood qualified as doctor (BMedSci, BMBS) from Nottingham University Medical School, Nottingham, UK, in 1999. She holds medical fellowships with the Royal College of Physicians (UK) and the Royal Australasian College of Physicians (Australia). She is a consultant respiratory physician in the Department of Respiratory Medicine at the Alfred Hospital (Melbourne, Australia), head of the Chronic Respiratory Disease Research Lab at the Central Clinical School (Monash University, Melbourne, Australia) and principal research fellow (associate professor) at Monash University (Australia). In addition to her qualifications in respiratory care, she got postgraduate qualifications in Palliative Care and Epidemiology (MSc; University of Melbourne, 2011) and a PhD (University of Melbourne, 2019). Assistant Professor Smallwood holds various major research grants including an NHMRC Investigator grant and fellowship research grant from the Windermere foundation. She has clinical and research interests in severe lung disease. As a frontline health worker, she designed and co-led the Australian COVID-19 Frontline Healthcare Workers study, which is the largest multi-professional, national study that has examined the psychosocial effects of the pandemic on healthcare workers in primary and secondary care. She is a board director for the Thoracic Society of Australia and New Zealand (TSANZ), the Victorian TSANZ branch president and serves on multiple national committees for TSANZ, the Lung Foundation Australia and the Palliative Care Clinical Studies Collaborative. In addition, she is a taskforce member for various national and international respiratory guidelines. 